\title{
Análisis de Eficiencia en Sistemas de Cómputo de Alta Performance Reconfigurables
}

\section{Martín Morales}

Centro de Técnicas Analógicos Digitales (CeTAD)

Universidad Nacional de La Plata / Universidad Nacional Arturo Jauretche, Argentina martin.morales@unaj.edu.ar

\section{Eduardo Kunysz}

Centro de Técnicas Analógicos Digitales (CeTAD)

Universidad Nacional de La Plata / Universidad Nacional Arturo Jauretche, Argentina ekunysz@unaj.edu.ar

\section{Jorge R. Osio}

Centro de Técnicas Analógicos Digitales (CeTAD)

Universidad Nacional de La Plata / Universidad Nacional Arturo Jauretche, Argentina josio@unaj.edu.ar

\section{José A. Rapallini}

Centro de Técnicas Analógicos Digitales (CeTAD)

Universidad Nacional de La Plata / Universidad Nacional Arturo Jauretche, Argentina jrapallini@gmail.com

Presentación 11/2016.

Aprobación 07/2017

\section{Resumen}

El objetivo de este trabajo, es presentar la arquitectura y características de un sistema para el estudio de las nuevas técnicas de procesamiento paralelo en el desarrollo de sistemas de cómputo de aplicaciones específicas de altas prestaciones. Como opción en la optimización de rendimiento y reducción en los consumos energéticos se propone una alternativa que representa un nuevo paradigma en el desarrollo de supercomputadoras. Dicha alternativa, viene de la mano de tecnologías de arreglos de compuertas lógicas formando parte del cómputo de alta performance reconfigurable (HPCR).

Palabras claves: HPRC, FPGA, Computación Paralela 


\begin{abstract}
The purpouse of this project is to introduce the architectures and characteristics for new technics of high performance computing paralleling process. As an option of optimization, performance and high energy efficient is propose a new paradigm in supercomputers design. This alternative has came with logic array technology as a part of high performance reconfigurable computer (HPRC).
\end{abstract}

Keywords: HPRC, FPGA, Parallel Computing

\title{
Introducción
}

A medida que la teoría de la información avanza, los desafíos en cuanto a capacidad de cómputo y cantidad de datos aumenta drásticamente. Hoy en día es algo normal el pensar en múltiples procesadores para la industria masiva y supercomputadoras para usos específicos.

Muchos investigadores han demostrado que la computadora de propósitos generales con procesadores gráficos (GPUs), son una alternativa interesante para resolver problemas de cálculos intensivos. El desafío en estos casos consiste en trasladar el problema concreto a las limitaciones de la programación gráfica. Éstos GPUs (Graphics Processing Unit), contienen múltiples procesadores simples, en su conjunto llamados many-cores. Este tipo de tecnología normalmente utiliza arquitecturas híbridas, donde parte del procesamiento se realiza en la computadora de propósitos generales y la otra en la placa gráfica.

Con la intención de expandir este concepto hacia aplicaciones más específicas dejando de lado la versatilidad, los fabricantes de computadoras de altas prestaciones, han introducido unidades de co-procesamiento de arreglos de lógica programable. La aplicación principal se ejecuta en los microprocesadores, mientras que las FPGAs manejan las porciones de código que requieren mayor tiempo de ejecución. Estas porciones de procesamiento suelen ser datos en paralelo superpuestos, arquitecturas que se pueden implementar con una alta granularidad, una sola instrucción, una instrucción y múltiples datos (SIMD), entre otros [2].

Una de las ventajas de este tipo de ar- quitectura es la capacidad que tiene el procesador principal de reconfigurar las FPGAs en tiempo real. Esto permite la reutilización de partes de hardware en virtud de mejorar la performance del software, lo que crea un nuevo paradigma en el campo de los desarrolladores de aplicaciones. Los sistemas tradicionales utilizan técnicas de descripción de hardware como VHDL. Otros utilizan lenguajes de alto nivel adaptados ([7-10]) como C o Fortran, o entornos de programación gráficos ([11] y [12]). Esas últimas alternativas permiten una transición natural entre el mundo del hardware y el software.

Con las primeras FPGAs, el reducido tamaño y capacidad que tenían, limitaba mucho la evolución de sistemas tan complejos como los que se pueden implementar en una ASIC. Pero hoy en día sabemos que los recursos de los dispositivos de arreglos de lógica programable se incrementan cada año debido a la Ley de Moore, con el agregado de memoria RAM embebida. Con estos incrementos se logran diseños de gran magnitud. Hasta tal punto es así, que la tendencia actual es integrar 
dentro de una FPGA sistemas digitales completos que incluyen un microprocesador de propósito general y todo el hardware de propósito específico que requiere la aplicación [1]

Esto ha llevado a elevar el número de integrados de lógica programable en comparación con el número de procesadores. Con lo que hoy en día tenemos plataformas de altas prestaciones en donde casi todo el procesamiento se realiza en FPGAs.

Típicamente el clock de una FPGA es un orden de magnitud más lento que el de un procesador. Sin embargo desde el punto de vista de la velocidad, las FPGAs obtienen su ventaja en los siguientes tres factores:

Intensidad: en el mejor de los casos un CPU puede realizar una operación entre enteros cada dos ciclos de reloj (caso ideal en donde se estaría utilizando cache, y con el pipeline funcionando sin interrupciones). El peor caso es significativamente más desfavorable. En una FPGA se implementa sólo la funcionalidad necesaria para la aplicación particular. Por lo tanto se prescinde del "overhead" producto de la arquitectura sofisticada que tiene un CPU de propósitos generales.

2. Latencia: con una FPGA se puede obtener una granularidad fina sobre el control de donde se encuentran los datos en memoria.

3. Paralelismo espacial: se puede realizar un pequeño pipeline de propósitos especiales para realizar una operación particular, y luego replicarlo dentro del chip de la FPGA.

Entre algunas de las alternativas que se pueden explorar con computadoras paralelas reconfigurables se tienen tres parámetros:

- Comunicación: posibilidad de ensayar diversas topologías conocidas, o explorar nuevas alternativas que podrían optimizar el rendimiento general. Los dispositivos más modernos permiten interfaces de alta velocidad como GbEthernet, o PCI Express. Se pueden implementar protocolos de comunicación flexibles y variar el ancho de bus según la necesidad. Una de las características que se puede utilizar con esta tecnología es la capacidad de reconfiguración parcial de los dispositivos de lógica programable. Esto último permite crear redes virtuales reconfigurables en hardware en tiempo real, que sirvan de soporte para el enrutado en software [13].

- Memoria: cada problema específico requiere de una configuración de memoria determinada. Para ello se cuenta con memoria interna (en general limitada) la cual se podría utilizar para procesos locales y luego se puede interconectar con distintas tecnologías de memoria existentes. Los dispositivos más modernos tienen incorporados módulos de control para memorias del tipo DDR3.

- Software: en este tipo de plataformas es el aspecto más difícil de estandarizar. El software necesariamente es híbrido entre partes de hardware (manejado por drivers) y lenguajes de alto nivel. En estos casos el desafío es encontrar que partes de alto nivel son las que generan mayor "overhead" sobre el procesamiento, identificarlas y luego trasladarlas a su versión de compuertas lógicas. (Implementarlas dentro de la FPGA)

Si bien el estudio de sistemas paralelos con múltiples procesadores, es una ciencia bien estudiada, el estudio de utilización de múltiples dispositivos reconfigurables no es un terreno completamente explorado. 


\section{Implementación}

El grupo de investigación está explorando técnicas de procesamiento de HPRC con una placa que está compuesta por una FPGA (Xilinx Spartan 6), 3 memorias SRAM para maximizar el throughput de acceso aleatorio y 2 memorias del tipo DDR2 para almacenamiento de datos. Esto, junto a las fuentes de alimentación e interfaces de comunicación están integrados en un circuito impreso de 12 capas y de un tamaño de $8 \times 12 \mathrm{~cm}$.

Sobre esta placa se está trabajando en el diseño de una maqueta de simulación para evolucionar hacia tecnologías superiores con varias FPGAs por placa.

En una primera etapa se trabajará sobre una unidad de procesamiento (UP) compuesta por dos placas de las presentadas.

En la Fig 1, se observa la arquitectura propuesta para esta variante.

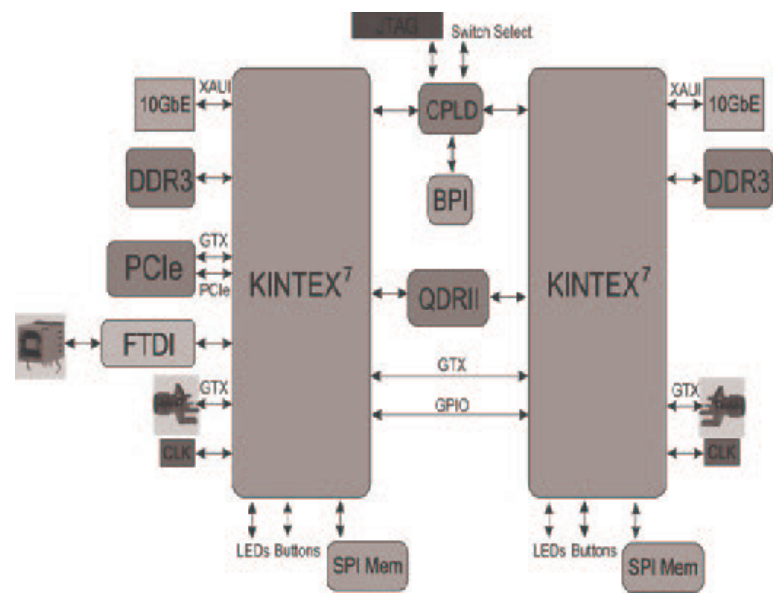

Fig. 1.Topología Backplane / Gigabit ethernet

Algunas de las características de este diseño son:

- Memorias DDR3

- SRAM Quad Data Rate (QDRII); memorias de alta velocidad y baja latencia.

- Memorias Flash BPI (Byte Peripheral Interface); memorias de configuración de alta velocidad.

- Interfaz integrada PCIe (Gen $2.05 \mathrm{~GB} / \mathrm{s}$ ), a través de los transceivers GTX.

- $10 \mathrm{~Gb}$ Ethernet, a través del protocolo XAUI (10 Attachment Unit Interface)

El objetivo es probar distintas opciones y topologías que puedan ser útiles en una versión más avanzada de 4 FPGAs por UP.

Para una etapa posterior, se estudian distintas topologías de interconexión que permitan flexibilidad de proyectos y aplicaciones específicas. 


\section{Simulaciones}

Uno de los grandes beneficios de la utilización de sistemas de arreglos de lógica programable es la gran versatilidad a la hora de simular comportamientos.

Existe un abanico enorme de aplicaciones de simulación, las cuales logran resultados no sólo adecuados, sino muy realistas del algoritmo.

Mediante dichas simulaciones no solo se puede verificar el flujo de la información sinó que tambiés en posible verificar los "constrains" temporales del circuito integrado.

Uno de los primeros trabajos que está realizando el grupo de investigación es la comparación a nivel de simulación de diversos algoritmos corriendo en una FPGA.

\section{Conclusiones}

La tecnología FPGA está introduciéndose fuertemente en el procesamiento paralelo. Se presenta como una alternativa fuerte y confiable a la resolución de varios problemas de la ciencia como de la industria.

Como se analizó, las computadoras de alta performance son necesarias en la resolución de cálculos con gran densidad de parámetros y datos. $\mathrm{Y}$ también para la búsqueda de información dentro de grandes bases de datos en donde se pueden implementar algoritmos de búsqueda por correlación [14].

Cada implementación o arquitectura está ligada directamente a la aplicación para la cual se desea implementar:

Teniendo en cuenta el nivel de comuni-cación se pueden utilizar topologías "backplane" (para requerimientos más leves) o topologías "cubo" para sistemas que presentan requisitos altos de comunicación y modularidad.

Teniendo en cuenta la simetría de los procesos, si se puede paralelizar en partes idénticas, tanto la tecnología como la comunicación es homogénea. Sin embargo, si el problema se puede paralelizar en partes diferentes, se puede pensar en sistemas heterogéneos, utilizando diferentes tecnologías de procesamiento por cada etapa.

Las necesidades comerciales y la disminución de riesgos al desarrollar una supercomputadora, hacen de las supercomputadoras basadas en tecnologías FPGA una apuesta interesante de mercado. 


\section{Referencias}

Mencer, O., Tsoi, K. H., Craimer, S., Todman, T., Wayne, L., Wong, M. Y., Leong, P. H. (s/d). "CUBE: a 512-FPGA Cluster" en IEEE Conference. Dept. of Computing, Imperial College London. Dept. of Computer Science and Engineering The Chinese University of Hong Kong .

Rupp, C., Landguth, M., Garverick, T., Gomersall, E., Holt, H. (s/d). “The NAPA Adaptive Processing Architecture" en IEEE Conference. National Semiconductor Corporation.

Makino, J., Taiji, M., Ebisuzaki, T., Sugimoto, D. (s/d). "grape-4 : A Massively Parallel Special-Purpose Computer for Collisional N-Body Simulations” en IEEE Conference.

Fidanci, O., Diab, H., El-Ghazawi, T., Gaj, K., Alexandridis, N. (s/d). "Implementation trade-offs of Triple DES in the SRC-6e Reconfigurable Computing Environment".

Underwood, K. (s/d). "FPGAs vs. CPUs: Trends in Peak Floating-Point Performance ", Sandia National Laboratories .

McMahon, P. L. (s/d)."High Performance Reconfigurable Computing for Science and Engineering Applications ”.

Meixner, D., Kindratenko, V., Pointer, D. (s/d)."Implementing Simulink Designs on SRC-6 System", Innovative Systems Laboratory, National Center for Supercomputing Applications, University of Illinois at Urbana-Champaign. Disponible en <http://www.srccomp.com/carte-programming-environment>.

Bodenner, R. (s/d). "Using Hardware Libraries with Impulse C", Director of Product Development, Impulse Accelerated Technologies. Application Note. Disponible en <http://www.impulseaccelerated.com>.

"Low Power Hybrid Computing for Efficient Software Acceleration" Mitrionics, White Paper. Disponible en <http://www.mitrionics.com>.

"DK4 - Handel-C Language Reference Manual", Celoxica. Disponible en <http:// www.celoxica.com>.

"CoreFireTM Design Suite", Annapolis Micro Systems, DataSheet. Disponible en <http://www.annapmicro.com/corefire.html>.

Kintali, K., Gu, Y. "Model-Based Design with Simulink, HDL Coder, and Xilinx System Generator for DSP". MathWorks, White Paper. Disponible en < http://www. mathworks.com/fpga-design/simulink-with-xilinx-system-generator-for-dsp. html>.

Yin, D., Unnikrishnan, D., Liao, Y., Gao, L., Tessier, R. (s/d). "Customizing Virtual Networks with Partial FPGA Reconfiguration". Dept. of Electrical and Computer Engineering University of Massachusetts.

Benkrid, K., Akoglu, A., Ling, C., Song, Y., Liu, Y., Tian, X. (2012). "High Performance Biological Pairwise Sequence Alignment: FPGA versus GPU versus Cell BE versus GPP”. International Journal of Reconfigurable Computing. 